\title{
Classification of solitary wave bifurcations in generalized nonlinear Schrödinger equations
}

\author{
Jianke Yang \\ Department of Mathematics and Statistics \\ University of Vermont \\ Burlington, VT 05401, USA
}

\begin{abstract}
Bifurcations of solitary waves are classified for the generalized nonlinear Schrödinger equations with arbitrary nonlinearities and external potentials in arbitrary spatial dimensions. Analytical conditions are derived for three major types of solitary wave bifurcations, namely saddlenode bifurcations, pitchfork bifurcations and transcritical bifurcations. Shapes of power diagrams near these bifurcations are also obtained. It is shown that for pitchfork and transcritical bifurcations, their power diagrams look differently from their familiar solution-bifurcation diagrams. Numerical examples for these three types of bifurcations are given as well. Of these numerical examples, one shows a transcritical bifurcation, which is the first report of transcritical bifurcations in the generalized nonlinear Schrödinger equations. Another shows a power loop phenomenon which contains several saddle-node bifurcations, and a third example shows double pitchfork bifurcations. These numerical examples are in good agreement with the analytical results.
\end{abstract}

\section{Introduction}

Solitary waves are spatially localized and temporally stationary (or steadily moving) solutions of nonlinear wave equations. Solitary waves play an important role in the understanding of nonlinear wave dynamics and thus have been heavily studied for a wide range of nonlinear wave models arising in diverse physical disciplines [1, 2]. When the propagation constant of solitary waves or physical parameters in the nonlinear wave equations changes, bifurcations of solitary waves can occur. Indeed, various solitary wave bifurcations in miscellaneous nonlinear wave models have been reported. Examples include saddle-node bifurcations (also called fold bifurcations) [2, 3, 4, 5, 6, 7, 8, 9, 10, 11], pitchfork bifurcations (sometimes called symmetry-breaking bifurcations) [11, 12, 13, 14, 15, 16, 17, 18, 19, transcritical bifurcations [7], and so on. Most of these reports on bifurcations are numerical. In the few analytical studies, focus was on the quantitative prediction of symmetry-breaking bifurcation points in the nonlinear Schrödinger (NLS) equations with symmetric double-well potentials [13, 15, 16, 18, 19] and the prediction of saddle-node and pitchfork bifurcation points in the NLS equations with periodic potentials [11. A general treatment of these bifurcations and general analytical conditions for their occurrences are still lacking at this time. 
In this paper, we systematically classify solitary wave bifurcations in the generalized NLS equations with arbitrary nonlinearities and external potentials in arbitrary spatial dimensions. These generalized NLS equations include the Gross-Pitaevskii equations in Bose-Einstein condensates [20] and nonlinear light-transmission equations in refractive-index-modulated optical media [1, 2] as special cases. For this large class of wave equations, we derive sufficient analytical conditions for three major types of solitary wave bifurcations, namely saddle-node bifurcations, pitchfork bifurcations and transcritical bifurcations. In addition, shapes of power diagrams near these bifurcation points are also derived. We will show that the power diagram near a saddle-node bifurcation is a horizontally oriented parabola; the power diagram near a pitchfork bifurcation is an extra power curve bifurcating out from a smooth power curve on one side of the bifurcation point (like a slanted letter ' $y$ '); and the power diagram near a transcritical bifurcation comprises two smooth curves tangentially connected at the bifurcation point. These analytical results are followed by various numerical examples. One example shows a transcritical bifurcation, which is the first report of transcritical bifurcations in the generalized NLS equations to the author's best knowledge. Another example shows double pitchfork bifurcations combined with saddle-node bifurcations, and a third example shows a power loop phenomenon which contains a number of saddle-node bifurdations. These numerical examples of bifurcations are found to be in good agreement with our analytical results.

\section{Preliminaries}

We consider the generalized nonlinear Schrödinger (GNLS) equations with arbitrary forms of nonlinearity and external potentials in any spatial dimensions. These equations can be written as

$$
i U_{t}+\nabla^{2} U+F\left(|U|^{2}, \mathbf{x}\right) U=0
$$

where $\nabla^{2}=\partial^{2} / \partial x_{1}^{2}+\partial^{2} / \partial x_{2}^{2}+\cdots+\partial^{2} / \partial x_{N}^{2}$ is the Laplacian in the $N$-dimensional space $\mathbf{x}=$ $\left(x_{1}, x_{2}, \cdots, x_{N}\right)$, and $F(\cdot, \cdot)$ is a general real-valued function which includes nonlinearity as well as external potentials. These GNLS equations are physically important since they include the GrossPitaevskii equations in Bose-Einstein condensates [20] and nonlinear light-transmission equations in refractive-index-modulated optical media [1, 2] as special cases. Notice that these GNLS equations are conservative and Hamiltonian.

For a large class of nonlinearities and potentials, these GNLS equations admit stationary solitary waves

$$
U(\mathbf{x}, t)=e^{i \mu t} u(\mathbf{x}),
$$

where $u(\mathbf{x})$ is a real localized function in the square-integrable functional space which satisfies the equation

$$
\nabla^{2} u-\mu u+F\left(u^{2}, \mathbf{x}\right) u=0,
$$

and $\mu$ is a real-valued propagation constant. Examples of such solitary waves can be found in numerous books and articles (see [1, 2] for instance). In these solitary waves, $\mu$ is a free parameter, and $u(\mathbf{x})$ depends continuously on $\mu$. Under certain conditions, these solitary waves undergo bifurcations at special values of $\mu$. Reported examples of bifurcations in Eq. (2.3) include saddlenode bifurcations [2, 8, 9, 11] and pitchfork bifurcations [11, 13, 14, 15, 16, 17, 18, 19]. Transcritical 
bifurcations in this equation have not been reported yet (even though they have been found in other nonlinear wave models [7]).

For later analysis, we introduce the linearization operator of Eq. (2.3),

$$
L_{1}=\nabla^{2}-\mu+\partial_{u}\left[F\left(u^{2}, \mathbf{x}\right) u\right]
$$

which is a self-adjoint linear Schrödinger operator. We also introduce the standard inner product of functions,

$$
\langle f, g\rangle=\int_{-\infty}^{\infty} f^{*}(\mathbf{x}) g(\mathbf{x}) d \mathbf{x} .
$$

In addition, we define the power of a solitary wave $u(\mathbf{x} ; \mu)$ as

$$
P(\mu)=\langle u, u\rangle=\int_{-\infty}^{\infty} u^{2}(\mathbf{x} ; \mu) d \mathbf{x}
$$

This power function not only conveniently characterizes solitary-wave families, but also plays an important role in the stability of these waves [2].

Our analysis of bifurcations starts with the basic observation that, if a bifurcation occurs at $\mu=\mu_{0}$, by denoting the corresponding solitary wave and the linearization operator as

$$
u_{0}(\mathbf{x})=u\left(\mathbf{x} ; \mu_{0}\right), \quad L_{10}=\left.L_{1}\right|_{\mu=\mu_{0}, u=u_{0}},
$$

then the linear operator $L_{10}$ should have a discrete zero eigenvalue. This is a necessary condition for bifurcations, hence it can be used to determine where a bifurcation might occur. This condition is not sufficient though. Indeed, if the function $F\left(|U|^{2}, \mathbf{x}\right)$ in (2.1) does not depend explicitly on a certain spatial dimension $x_{j}$, i.e., the GNLS equation (2.1) is translation-invariant along the $x_{j^{-}}$ dimension, then for any solitary wave $u(\mathbf{x} ; \mu), L_{1} u_{x_{j}}=0$, i.e., $L_{1}$ has a discrete zero eigenvalue. But this zero eigenvalue of $L_{1}$ only corresponds to a spatial translation of $u(\mathbf{x} ; \mu)$ and does not imply solitary wave bifurcations. More will be said on this issue in the later text (see Remark 3 in Sec. [3).

In the next section, we will derive sufficient conditions for three major types of solitary wave bifurcations. To simplify the analysis, we will focus on the case where this zero eigenvalue of $L_{10}$ is simple. Hence we introduce the following assumption.

Assumption 1 Suppose at a certain propagation constant $\mu=\mu_{0}, L_{10}$ has a zero eigenvalue. Then it is assumed that this zero eigenvalue of $L_{10}$ is simple and discrete.

This assumption is satisfied for almost all one-dimensional bifurcations and many higherdimensional bifurcations. The case of $L_{10}$ 's zero eigenvalue being multi-fold (repeated) can be similarly treated, and that will be done elsewhere.

Remark 1 Due to Assumption 1, the zero eigenvalue of $L_{10}$ is simple and discrete, thus this zero eigenvalue is not embedded inside the continuous spectrum of $L_{10}$. This means that the solitary wave $u_{0}(\mathbf{x})$ at $\mu=\mu_{0}$ is not an embedded soliton [2]. This fact allows us to construct solitary waves in the vicinity of $\mu=\mu_{0}$ by perturbation series expansions without worrying about continuous-wave tails beyond all orders of the perturbation expansion [2, 21, 22, 23]. 
Under Assumption 1, we denote the single discrete (localized) eigenfunction of $L_{10}$ at the zero eigenvalue as $\psi(\mathbf{x})$, i.e.,

$$
L_{10} \psi=0 .
$$

Since $L_{10}$ is a real operator, the eigenfunction $\psi$ can be normalized to be a real function. Thus $\psi$ will be taken as a real function in the remainder of this article. We also denote

$$
G(u ; \mathbf{x})=F\left(u^{2} ; \mathbf{x}\right) u, \quad G_{k}(\mathbf{x})=\left.\partial_{u}^{k} G\right|_{u=u_{0}}, k=0,1,2,3, \ldots
$$

These notations will be used in the next sections.

\section{The main results}

In this section, we present sufficient analytical conditions for three major types of solitary wave bifurcations, namely, the saddle-node bifurcation, the pitchfork bifurcation, and the transcritical bifurcation. In addition, power diagrams of these solitary waves near bifurcation points will also be described.

First we explain what these three bifurcations are. A saddle-node bifurcation is where on one side of the bifurcation point $\mu_{0}$, there are no solitary wave solutions; but on the other side of $\mu_{0}$, there are two distinct solitary wave branches. These two branches merge with each other as $\mu \rightarrow \mu_{0}$. This bifurcation is also called a fold bifurcation in the literature (following a similar practice in dynamical systems [24]). Examples of this bifurcation in the GNLS equation (2.3) can be found in $\left[2,8,9,11\right.$. A pitchfork bifurcation is where on one side of the bifurcation point $\mu_{0}$, there is a single solitary wave branch; but on the other side of $\mu_{0}$, there are three distinct solitary wave branches. One of these three branches is a smooth continuation of the single solution branch from the other side of $\mu_{0}$, but the other two branches are new and they bifurcate out at the bifurcation point $\mu_{0}$. As $\mu \rightarrow \mu_{0}$, these two new solution branches merge with the smooth branch. Examples of pitchfork bifurcations reported so far are all symmetry-breaking bifurcations [11, 13, 14, 15, 16, 17, 18, 19], where a smooth branch of symmetric or antisymmetric solitary waves exists on both sides of the bifurcation point, but two new branches of asymmetric solutions appear on only one side of the bifurcation point. A transcritical bifurcation is where there are two smooth branches of solitary waves which exist on both sides of the bifurcation point $\mu_{0}$, and these solutions on both branches approach each other as $\mu \rightarrow \mu_{0}$. So far, no examples of transcritical bifurcations of solitary waves have been reported in the GNLS equation (2.3) yet (to the author's best knowledge). But these transcritical bifurcations do exist in Eq. (2.3), and one such example will be presented in Sec. 5 of this article.

The main result of this article is the following theorem which gives sufficient analytical conditions for the above three major types of solitary wave bifurcations.

Theorem 1 Under Assumption 1, the following three statements hold.

1. If $\left\langle u_{0}, \psi\right\rangle \neq 0$ and $\left\langle G_{2}, \psi^{3}\right\rangle \neq 0$, then a saddle-node bifurcation occurs at $\mu=\mu_{0}$. When these two non-zero quantities have the same (opposite) sign, the solutions bifurcate to the right (left) side of $\mu=\mu_{0}$. 
2. If $\left\langle u_{0}, \psi\right\rangle=\left\langle G_{2}, \psi^{3}\right\rangle=0,\left\langle 1-G_{2} L_{10}^{-1} u_{0}, \psi^{2}\right\rangle \neq 0$, and $\left\langle G_{3}, \psi^{4}\right\rangle-3\left\langle G_{2} \psi^{2}, L_{10}^{-1}\left(G_{2} \psi^{2}\right)\right\rangle \neq 0$, then a pitchfork bifurcation occurs at $\mu=\mu_{0}$. When these two non-zero quantities have the same (opposite) sign, the new solution branches bifurcate to the right (left) side of $\mu=\mu_{0}$.

3. If $\left\langle u_{0}, \psi\right\rangle=0,\left\langle G_{2}, \psi^{3}\right\rangle \neq 0$, and

$$
\left\langle 1-G_{2} L_{10}^{-1} u_{0},, \psi^{2}\right\rangle^{2}>\left\langle G_{2}, \psi^{3}\right\rangle\left\langle G_{2}\left(L_{10}^{-1} u_{0}\right)^{2}-2 L_{10}^{-1} u_{0}, \psi\right\rangle,
$$

then a transcritical bifurcation occurs at $\mu=\mu_{0}$.

It is noted that under the conditions of cases 2 and 3 in this theorem, real quantities $L_{10}^{-1} u_{0}$ and $L_{10}^{-1}\left(G_{2} \psi^{2}\right)$, which appear in these conditions, exist (see Lemma 1 in the next section).

Theorem 1 shows that in the generic case of $\left\langle u_{0}, \psi\right\rangle \neq 0$ and $\left\langle G_{2}, \psi^{3}\right\rangle \neq 0$, a saddle-node bifurcation occurs. Pitchfork and transcritical bifurcations would arise only in more restrictive situations. For instance, pitchfork bifurcations generally occur only in symmetric potentials, see [11, 13, 14, 15, 16, 17, 18, 19] and Remark 2 below. Transcritical bifurcations are more rare, which explains why they have not been found in Eq. (2.3) before. The above situation closely resembles that in finite-dimensional dynamical systems [25]. More will be said on this in the end of Sec. 5 .

Remark 2 An important (dominant) class of pitchfork bifurcations is the symmetry-breaking bifurcation. Suppose the potential in Eq. (2.1) is symmetric, i.e.,

$$
F\left(u^{2} ;-\mathbf{x}\right)=F\left(u^{2} ; \mathbf{x}\right) .
$$

In addition, suppose the solitary wave $u_{0}(\mathbf{x})$ has certain symmetry (even or odd in $\mathbf{x}$ ), and the eigenfunction $\psi(\mathbf{x})$ has the opposite symmetry of $u_{0}(\mathbf{x})$ (odd or even), i.e.,

$$
u_{0}(-\mathbf{x})= \pm u_{0}(\mathbf{x}), \quad \psi(-\mathbf{x})=\mp \psi(\mathbf{x}) .
$$

From the notation (2.9), we see that

$$
G_{2}=\left[6 u \partial_{u^{2}} F\left(u^{2} ; \mathbf{x}\right)+4 u^{3} \partial_{u^{2}}^{2} F\left(u^{2} ; \mathbf{x}\right)\right]_{u=u_{0}},
$$

which has the same symmetry as $u_{0}(\mathbf{x})$. Then obviously,

$$
\left\langle u_{0}, \psi\right\rangle=\left\langle G_{2}, \psi^{3}\right\rangle=0,
$$

thus the conditions of Case 2 in Theorem 1 are generically satisfied. Consequently, a pitchfork bifurcation occurs at $\mu=\mu_{0}$. In this case, the two bifurcated solutions $u^{ \pm}(\mathbf{x} ; \mu)$ are simply related as

$$
u^{-}(\mathbf{x} ; \mu)=u^{+}(-\mathbf{x} ; \mu)
$$

In addition, these bifurcated solutions break the symmetry of the original $u_{0}(\mathbf{x})$ solution and are asymmetric, as can be seen from their asymptotic solution formulae in Eq. (4.46) later. This explains why this pitchfork bifurcation is often called symmetry-breaking bifurcation in the literature. To the author's knowledge, all pitchfork bifurcations reported so far are symmetry-breaking bifurcations.

Remark 3 Suppose Eq. (2.1) is translation invariant along a certain spatial dimension $x_{j}$, i.e., $F\left(|U|^{2}, \mathbf{x}\right)$ in (2.1) does not depend explicitly on $x_{j}$. If this equation admits a solitary wave $u_{0}(\mathbf{x})$ 
at $\mu=\mu_{0}$, then by differentiating Eq. (2.3) with respect to $x_{j}$, we find that $L_{10} u_{0, x_{j}}=0$, thus zero is a discrete eigenvalue of $L_{10}$ with eigenfunction $\psi=u_{0, x_{j}}$. In this case, simple calculations show that

$$
\left\langle u_{0}, \psi\right\rangle=\left\langle G_{2}, \psi^{3}\right\rangle=\left\langle 1-G_{2} L_{10}^{-1} u_{0}, \psi^{2}\right\rangle=0
$$

and

$$
\left\langle G_{3}, \psi^{4}\right\rangle-3\left\langle G_{2} \psi^{2}, L_{10}^{-1}\left(G_{2} \psi^{2}\right)\right\rangle=0 .
$$

Thus this case does not fall into any of the three cases in Theorem 1, hence no solitary wave bifurcation can be predicted. This is not surprising, since a zero eigenvalue induced by translation invariance does not create solitary wave bifurcations in general.

Power diagrams are important not only for displaying solitary wave bifurcations but also for predicting stability properties of these solitary waves [2]. The power diagrams near these three types of bifurcations are given in the following theorem.

Theorem 2 Suppose Assumption 1 holds. Denoting the power of the solitary wave at the bifurcation point as $P_{0}=\left\langle u_{0}, u_{0}\right\rangle$, then

1. near the saddle-node bifurcation in Case 1 of Theorem 1, power functions of the two solution branches $u^{ \pm}(\mathbf{x} ; \mu)$ are

$$
P^{ \pm}(\mu)=P_{0} \pm P_{1} \cdot\left(\mu-\mu_{0}\right)^{1 / 2}+O\left(\mu-\mu_{0}\right),
$$

where the constant $P_{1}$ is given by

$$
P_{1}=2\left\langle u_{0}, \psi\right\rangle \sqrt{\frac{2\left\langle u_{0}, \psi\right\rangle}{\left\langle G_{2}, \psi^{3}\right\rangle}} ;
$$

2. near the pitchfork bifurcation in Case 2 of Theorem 1, the power function for the smooth solution branch $u^{0}(\mathbf{x} ; \mu)$ is

$$
P^{0}(\mu)=P_{0}+P_{1}^{0} \cdot\left(\mu-\mu_{0}\right)+O\left\{\left(\mu-\mu_{0}\right)^{2}\right\},
$$

where the constant $P_{1}^{0}$ is given by

$$
P_{1}^{0}=2\left\langle u_{0}, L_{10}^{-1} u_{0}\right\rangle
$$

power functions for the two bifurcated solution branches $u^{ \pm}(\mathbf{x} ; \mu)$ are

$$
P^{ \pm}(\mu)=P_{0}+P_{1} \cdot\left(\mu-\mu_{0}\right)+O\left\{\left(\mu-\mu_{0}\right)^{3 / 2}\right\}
$$

where the constant $P_{1}$ is given by

$$
P_{1}=2\left\langle u_{0}, L_{10}^{-1} u_{0}\right\rangle+\frac{6\left\langle 1-G_{2} L_{10}^{-1} u_{0}, \psi^{2}\right\rangle^{2}}{\left\langle G_{3}, \psi^{4}\right\rangle-3\left\langle G_{2} \psi^{2}, L_{10}^{-1}\left(G_{2} \psi^{2}\right)\right\rangle} ;
$$


3. near the transcritical bifurcation in Case 3 of Theorem 1, power functions for the two solution branches are

$$
P^{ \pm}(\mu)=P_{0}+P_{1} \cdot\left(\mu-\mu_{0}\right)+P_{2}^{ \pm} \cdot\left(\mu-\mu_{0}\right)^{2}+O\left\{\left(\mu-\mu_{0}\right)^{3}\right\}
$$

where the constants $P_{1}$ and $P_{2}^{ \pm}$are given by

$$
P_{1}=2\left\langle u_{0}, L_{10}^{-1} u_{0}\right\rangle, \quad P_{2}^{ \pm}=2\left\langle u_{0}, \widehat{u}_{2}^{ \pm}\right\rangle+\left\langle u_{1}^{ \pm}, u_{1}^{ \pm}\right\rangle,
$$

with $u_{1}^{ \pm}$specified in Eq. (4.58), $\widehat{u}_{2}^{ \pm}$being particular solutions to Eq. (4.59), and $b_{1}$ in (4.58)-(4.59) given in (4.61).

This theorem shows that the power diagram near a saddle-node bifurcation is a horizontally oriented parabola. Near a pitchfork bifurcation, power curves of the three solution branches are all linear functions of $\mu$. In addition, the two bifurcated solution branches $u^{ \pm}(\mathbf{x} ; \mu)$ have the same power slope at the bifurcation point. In fact, in the dominant case of symmetry-breaking bifurcations discussed in Remark 2, power curves $P^{ \pm}(\mu)$ of the two solution branches $u^{ \pm}(\mathbf{x} ; \mu)$ are identical for all $\mu$ both near and not near the bifurcation point, i.e., $P^{+}(\mu) \equiv P^{-}(\mu)$, due to the relation (3.3). It is also important to notice that the smooth solution branch $u^{0}(\mathbf{x} ; \mu)$ and the bifurcated solution branches $u^{ \pm}(\mathbf{x} ; \mu)$ have different power slopes at the bifurcation point, i.e., $P_{1}^{0} \neq P_{1}$, because the numerator in the second term of Eq. (3.9) is nonzero for pitchfork bifurcations (see Theorem 1). Thus, the power diagram near a pitchfork bifurcation looks like a slanted letter ' $y$ '. Near a transcritical bifurcation, power slopes of the two solution branches at the bifurcation point are the same, but their curvatures are different in the generic case. Thus the power diagram near a transcritical bifurcation comprises two smooth curves tangentially connected at the bifurcation point. These features of the power diagrams (for pitchfork and transcritical bifurcations) differ significantly from their familiar solution-bifurcation diagrams, and these differences are illustrated schematically in Fig. 1.

The upper row of this figure plots the deviation values $u\left(\mathbf{x}_{0} ; \mu\right)-u_{0}\left(\mathbf{x}_{0}\right)$, as a function of $\mu$, between solitary waves $u(\mathbf{x} ; \mu)$ away from the bifurcation point and the solitary wave $u_{0}(\mathbf{x})$ at the bifurcation point at a representative (fixed) $\mathbf{x}_{0}$ position. These curves are drawn using the leadingorder perturbation series solutions (4.12) (for the saddle-node bifurcation), (4.23) and (4.46) (for the pitchfork bifurcation), and (4.68) (for the transcritical bifurcation), which we will derive in the next section. Notice that these deviation diagrams closely resemble the corresponding bifurcation diagrams (of the same names) in finite-dimensional dynamical systems [25]. The lower row of Fig. 1 plots the associated power diagrams for the bifurcations in the upper row. These power curves are drawn using the power-function's asymptotic formulae (3.4), (3.6), (3.8) and (3.10) in Theorem 2. Notice that the power diagram of the pitchfork bifurcation has a double-branching structure rather than the familiar triple-branching structure, and the power diagram of the transcritical bifurcation has a tangential-intersection structure rather than the familiar ' $\mathrm{x}$ '-like crossing structure. These power-diagram behaviors have no counterparts in finite-dimensional dynamical systems, and they should be borne in mind when identifying solitary wave bifurcations in the GNLS equations (2.1).

\section{Proofs of the main results}

To prove the main results in Theorems 1 and 2, the following lemma will be used. 

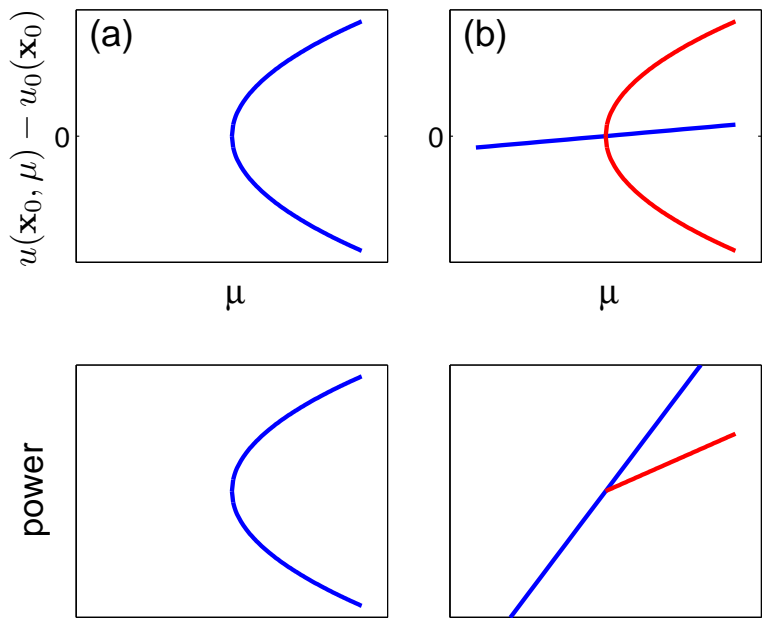

$\mu$ $\mu$

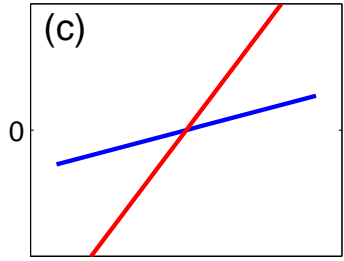

$\mu$

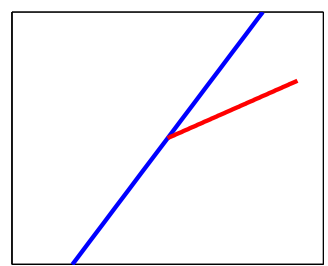

$\mu$

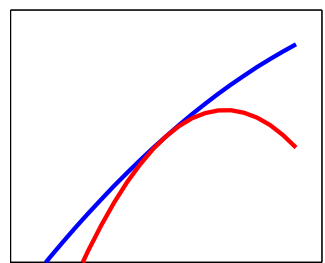

$\mu$

Figure 1: Schematic plots for solitary wave bifurcations (upper row) and the associated power diagrams (lower row). Column (a): saddle-node bifurcation; column (b): pitchfork bifurcation; column (c): transcritical bifurcation. The upper row shows the deviations $u\left(\mathbf{x}_{0} ; \mu\right)-u_{0}\left(\mathbf{x}_{0}\right)$ versus $\mu$ at a representative $\mathbf{x}_{0}$ position. These plots are drawn using the perturbation-series solution (4.12) for (a), (4.23) and (4.46) for (b), and (4.68) for (c). The power diagrams in the lower row are drawn using the asymptotic power-function formula (3.4) for (a), (3.6) and (3.8) for (b), and (3.10) for (c). Blue and red colors in columns (b,c) represent different solution branches.

Lemma 1 Suppose $f(\mathbf{x})$ is a localized function. Then under Assumption 1, the linear inhomogeneous equation

$$
L_{10} \phi=f
$$

admits localized solutions $\phi$ if and only if the inhomogeneous term $f$ is orthogonal to the homogeneous solution $\psi$, i.e.,

$$
\langle\psi, f\rangle=0
$$

This lemma is a direct consequence of the Fredholm Alternative Theorem. It can also be proved by expanding the localized function $f(\mathbf{x})$ and the solution $\phi(\mathbf{x})$ into the complete set of eigenfunctions of the Schrödinger operator $L_{10}$ and then solving for $\phi(\mathbf{x})$ directly.

In the later text, the orthogonality condition (4.2) will be called the solvability condition of the inhomogeneous equation (4.1) (for the existence of localized solutions).

Proof of Theorem 1 We will use the constructive method to prove this theorem. Specifically, we will explicitly construct solitary wave solutions, in the form of perturbation series expansions, which exist near $\mu=\mu_{0}$ under the conditions of this theorem. It will be shown that these perturbation series solutions can be constructed to all orders. The existence of available solitary wave solutions near $\mu=\mu_{0}$ will readily reveal the type of bifurcations at $\mu=\mu_{0}$.

\section{Case 1: saddle-node bifurcations}

Here we consider the first case of Theorem 1, and show that under its conditions $\left\langle u_{0}, \psi\right\rangle \neq 0$ 
and $\left\langle G_{2}, \psi^{3}\right\rangle \neq 0$, there exist two solitary-wave branches on only one side of $\mu=\mu_{0}$, which merge with each other as $\mu \rightarrow \mu_{0}$. We will also show that no other solitary wave solutions can be found near $\mu=\mu_{0}$. Hence a saddle-node bifurcation occurs here.

The solitary waves which exist near $\mu=\mu_{0}$ in this case have the following perturbation series expansions

$$
\begin{aligned}
u(\mathbf{x} ; \mu) & =\sum_{k=0}^{\infty}\left(\mu-\mu_{0}\right)^{k / 2} u_{k}(\mathbf{x}) \\
& =u_{0}(\mathbf{x})+\left(\mu-\mu_{0}\right)^{1 / 2} u_{1}(\mathbf{x})+\left(\mu-\mu_{0}\right) u_{2}(\mathbf{x})+\cdots .
\end{aligned}
$$

Inserting this expansion into Eq. (2.3) and recalling the notations (2.9), we get the following sequence of equations for $u_{k}$ at order $\left(\mu-\mu_{0}\right)^{k / 2}, k=0,1,2,3, \ldots$ :

$$
\begin{aligned}
& \nabla^{2} u_{0}-\mu_{0} u_{0}+F\left(u_{0}^{2}, \mathbf{x}\right) u_{0}=0, \\
& L_{10} u_{1}=0, \\
& L_{10} u_{2}=u_{0}-\frac{1}{2 !} G_{2} u_{1}^{2}, \\
& L_{10} u_{3}=u_{1}-G_{2} u_{1} u_{2}-\frac{1}{3 !} G_{3} u_{1}^{3}, \\
& L_{10} u_{4}=u_{2}-\frac{1}{2 !} G_{2}\left(u_{2}^{2}+2 u_{1} u_{3}\right)-\frac{1}{2 !} G_{3} u_{1}^{2} u_{2}-\frac{1}{4 !} G_{4} u_{1}^{4}, \\
& \ldots
\end{aligned}
$$

The equation (4.4) for $u_{0}$ is satisfied automatically since $u_{0}$ is a solitary wave of Eq. (2.3) at $\mu=\mu_{0}$. The $u_{1}$ solution to Eq. (4.5), under Assumption 1, is

$$
u_{1}=b_{1} \psi
$$

where $b_{1}$ is a constant. The $u_{2}$ function satisfies the linear inhomogeneous equation (4.6). Due to Lemma 1, Eq. (4.6) admits a localized solution for $u_{2}$ if and only if

$$
\left\langle\psi, u_{0}-\frac{1}{2} G_{2} u_{1}^{2}\right\rangle=0 .
$$

Inserting the $u_{1}$ solution (4.9) into this orthogonality condition and recalling the assumptions of Case 1, we find that

$$
b_{1}= \pm \eta, \quad \eta \equiv \sqrt{\frac{2\left\langle u_{0}, \psi\right\rangle}{\left\langle G_{2}, \psi^{3}\right\rangle}} .
$$

Thus, we get two $b_{1}$ solutions $\pm \eta$ which are opposite of each other. Inserting the corresponding $u_{1}$ solutions (4.9) into (4.3), we then get two perturbation series solutions of $u(\mathbf{x} ; \mu)$ as

$$
u^{ \pm}(\mathbf{x} ; \mu)=u_{0}(\mathbf{x}) \pm \eta\left(\mu-\mu_{0}\right)^{1 / 2} \psi(\mathbf{x})+O\left(\mu-\mu_{0}\right) .
$$

If $\left\langle u_{0}, \psi\right\rangle$ and $\left\langle G_{2}, \psi^{3}\right\rangle$ have the same sign, then $\eta$ is real. Recalling that $u_{0}(\mathbf{x})$ and $\psi(\mathbf{x})$ are both real localized functions, we see that these two perturbation series solutions (4.12) give two realvalued (legitimate) solitary waves when $\mu>\mu_{0}$, but not when $\mu<\mu_{0}$. On the other hand, if $\left\langle u_{0}, \psi\right\rangle$ 
and $\left\langle G_{2}, \psi^{3}\right\rangle$ have the opposite sign, $\eta$ is purely imaginary. In this case, the perturbation series solutions (4.12) give two real-valued solitary waves when $\mu<\mu_{0}$, but not when $\mu>\mu_{0}$.

Next we show that the two real localized perturbation series solutions (4.12), which exist on only one side of $\mu=\mu_{0}$, can be constructed to all orders of $\left(\mu-\mu_{0}\right)^{1 / 2}$. Let us first consider the $u_{2}$ equation (4.6). When $b_{1}$ is selected from Eq. (4.11), the orthogonality condition (4.10) is satisfied. Thus by Lemma 1, localized solutions for $u_{2}$ exist. Since the inhomogeneous term and the linear operator $L_{10}$ of (4.6) are both real, these localized $u_{2}$ solutions can also be made real. Let us denote one of such real localized $u_{2}$ solutions as $\widehat{u}_{2}$, i.e.,

$$
\widehat{u}_{2}=L_{10}^{-1}\left(u_{0}-\frac{1}{2} \eta^{2} G_{2} \psi^{2}\right)
$$

then since $\psi$ is a homogeneous localized solution of (4.6), the general localized solution of (4.6) is

$$
u_{2}=\widehat{u}_{2}+b_{2} \psi
$$

where $b_{2}$ is a constant to be determined.

Now we proceed to the $u_{3}$ equation (4.7). Inserting the $u_{1}$ and $u_{2}$ solutions (4.9) and (4.13) into (4.7), we get

$$
L_{10} u_{3}=b_{1}\left(\psi-G_{2} \widehat{u}_{2} \psi-\frac{1}{3 !} b_{1}^{2} G_{3} \psi^{3}-b_{2} G_{2} \psi^{2}\right) .
$$

By Lemma 1, this equation admits localized $u_{3}$ solutions if and only if its right hand side is orthogonal to the homogeneous solution $\psi$. Imposition of this orthogonality condition yields the $b_{2}$ value as

$$
b_{2}=\frac{\left\langle 1-G_{2} \widehat{u}_{2}-\eta^{2} G_{3} \psi^{2} / 3 !, \psi^{2}\right\rangle}{\left\langle G_{2}, \psi^{3}\right\rangle},
$$

which is a real constant. Notice that with this $b_{2}$ value, the solution $u_{2}(\mathbf{x})$ in (4.13) is the same for both choices $\pm \eta$ of $b_{1}$ in the $u_{1}$ solution (4.9), thus $u_{2}(\mathbf{x})$ is the same for both branches of the perturbation series solutions $u^{ \pm}(\mathbf{x} ; \mu)$ in (4.12). With the $b_{2}$ value (4.15), Eq. (4.14) admits localized solutions

$$
u_{3}=b_{1}\left(\widehat{u}_{3}+b_{3} \psi\right),
$$

where $\widehat{u}_{3}$ is a real-valued localized solution of Eq. (4.14) but without the $b_{1}$ factor on its right hand side, and $b_{3}$ is a constant. This $b_{3}$ will be determined from the solvability (orthogonality) condition of the $u_{4}$ equation (4.8) and can be found to be real. Note that $\widehat{u}_{3}(\mathbf{x})$ and $b_{3}$ are also the same for both branches of perturbation series solutions $u^{ \pm}(\mathbf{x} ; \mu)$.

Proceeding to higher orders and using the method of induction, we can readily show that all even terms $u_{2 n}$ are of the form

$$
u_{2 n}=\widehat{u}_{2 n}+b_{2 n} \psi, \quad n=1,2, \ldots,
$$

and all odd terms are of the form

$$
u_{2 n+1}=b_{1}\left[\widehat{u}_{2 n+1}+b_{2 n+1} \psi\right], \quad n=1,2, \ldots,
$$


where $\widehat{u}_{2 n}(\mathbf{x})$ and $\widehat{u}_{2 n+1}(\mathbf{x})$ are certain real localized functions, and $b_{2 n}, b_{2 n+1}$ are certain unique real constants. In addition, $u_{2 n}(\mathbf{x}), \widehat{u}_{2 n+1}(\mathbf{x})$, and $b_{2 n+1}$ are the same for both branches of perturbation series solutions $u^{ \pm}(\mathbf{x} ; \mu)$. Thus, by denoting $\widetilde{u}_{2 n+1}=\widehat{u}_{2 n+1}+b_{2 n+1} \psi$, we have

$$
u_{2 n}^{ \pm}=u_{2 n}(\mathbf{x}), \quad u_{2 n+1}^{ \pm}= \pm \eta \widetilde{u}_{2 n+1}(\mathbf{x})
$$

Inserting these $u_{2 n}^{ \pm}$and $u_{2 n+1}^{ \pm}$solutions into (4.3), we obtain two perturbation series solutions for $u(\mathbf{x} ; \mu)$, to all orders of $\left(\mu-\mu_{0}\right)^{1 / 2}$, as

$$
\begin{aligned}
u^{ \pm}(\mathbf{x} ; \mu)= & u_{0}(\mathbf{x})+\sum_{n=1}^{\infty}\left(\mu-\mu_{0}\right)^{n} u_{2 n}(\mathbf{x}) \\
& \pm \eta\left(\mu-\mu_{0}\right)^{1 / 2}\left\{\psi(\mathbf{x})+\sum_{n=1}^{\infty}\left(\mu-\mu_{0}\right)^{n} \widetilde{u}_{2 n+1}(\mathbf{x})\right\} .
\end{aligned}
$$

These two solutions exist on only one side of $\mu=\mu_{0}$ and are real and localized. The side of their existence depends on whether $\eta$ in (4.11) is real or imaginary. When $\mu \rightarrow \mu_{0}, u^{ \pm}(\mathbf{x} ; \mu) \rightarrow u_{0}(\mathbf{x})$, thus $u^{ \pm}(\mathbf{x} ; \mu)$ approach each other and merge at the bifurcation point.

Lastly, we show that except the above two solitary wave branches which exist on only one side of the bifurcation point, we can not find other solitary wave solutions near this bifurcation point. For instance, if we look for smooth solitary-wave branches which exist on both sides of $\mu=\mu_{0}$, then their perturbation expansions should be

$$
u(\mathbf{x} ; \mu)=u_{0}(\mathbf{x})+\left(\mu-\mu_{0}\right) u_{1}(\mathbf{x})+\left(\mu-\mu_{0}\right)^{2} u_{2}(\mathbf{x})+\cdots .
$$

When this expansion is substituted into (2.3), the $O(1)$ equation is still (4.4) which is satisfied. At $O\left(\mu-\mu_{0}\right)$, we get the equation for $u_{1}$ as

$$
L_{10} u_{1}=u_{0}
$$

Under conditions of Case 1, $\left\langle\psi, u_{0}\right\rangle \neq 0$. Thus by Lemma 1, Eq. (4.22) can not admit any localized solution for $u_{1}$. This means that solitary waves with the perturbation expansion (4.21) can not exist in this case. We have also searched solitary waves near $\mu=\mu_{0}$ in other perturbation series expansions, and could not find such solutions either. Thus a saddle-node bifurcation occurs at $\mu=\mu_{0}$.

\section{Case 2: pitchfork bifurcations}

Now we consider the second case of Theorem 1. We will show that under conditions of this case, a smooth branch of solitary waves exists on both sides of $\mu=\mu_{0}$. In addition, two other solitary-wave branches exist on only one side of $\mu=\mu_{0}$. As $\mu \rightarrow \mu_{0}$, all these solution branches approach the same solitary wave $u_{0}(\mathbf{x})$. Thus a pitchfork bifurcation occurs at $\mu=\mu_{0}$.

(i) We first construct the smooth branch of solitary waves which exists on both sides of $\mu=\mu_{0}$. These solitary waves have the following perturbation series expansion

$$
u^{0}(\mathbf{x} ; \mu)=\sum_{k=0}^{\infty}\left(\mu-\mu_{0}\right)^{k} u_{k}(\mathbf{x})
$$


Inserting this expansion into Eq. (2.3), we get the following sequence of equations for $u_{k}$ at orders $\left(\mu-\mu_{0}\right)^{k}, k=0,1,2,3, \ldots$ :

$$
\begin{aligned}
& \nabla^{2} u_{0}-\mu_{0} u_{0}+F\left(u_{0}^{2}, \mathbf{x}\right) u_{0}=0, \\
& L_{10} u_{1}=u_{0}, \\
& L_{10} u_{2}=u_{1}-\frac{1}{2 !} G_{2} u_{1}^{2}, \\
& L_{10} u_{3}=u_{2}-G_{2} u_{1} u_{2}-\frac{1}{3 !} G_{3} u_{1}^{3}, \\
& L_{10} u_{4}=u_{3}-\frac{1}{2 !} G_{2}\left(u_{2}^{2}+2 u_{1} u_{3}\right)-\frac{1}{2 !} G_{3} u_{1}^{2} u_{2}-\frac{1}{4 !} G_{4} u_{1}^{4},
\end{aligned}
$$

The equation (4.24) for $u_{0}$ is satisfied automatically. Under conditions of Case $2,\left\langle\psi, u_{0}\right\rangle=0$. Thus by Lemma 1, the solvability condition for the $u_{1}$ equation (4.25) is satisfied, hence this equation admits localized solutions

$$
u_{1}=\widehat{u}_{1}+b_{1} \psi
$$

where

$$
\widehat{u}_{1}=L_{10}^{-1} u_{0}
$$

is a real localized particular solution to Eq. (4.25), and $b_{1}$ is a constant to be determined. Inserting this $u_{1}$ solution into the $u_{2}$ equation (4.26), we get

$$
L_{10} u_{2}=\widehat{u}_{1}-\frac{1}{2} G_{2} \widehat{u}_{1}^{2}+b_{1} \psi\left(1-G_{2} \widehat{u}_{1}\right)-\frac{1}{2} b_{1}^{2} G_{2} \psi^{2} .
$$

By Lemma 1, the solvability condition of this $u_{2}$ equation is that its right hand side be orthogonal to the homogeneous solution $\psi$. Under conditions of Case $2,\left\langle G_{2}, \psi^{3}\right\rangle=0$. Thus this solvability condition gives

$$
b_{1}\left\langle 1-G_{2} L_{10}^{-1} u_{0}, \psi^{2}\right\rangle=\left\langle\frac{1}{2} G_{2} \widehat{u}_{1}^{2}-\widehat{u}_{1}, \psi\right\rangle .
$$

Since the inner product on the left side of this equation is nonzero under conditions of Case 2 , this equation yields a unique $b_{1}$ value as

$$
b_{1}=\frac{\left\langle G_{2} \widehat{u}_{1}^{2} / 2-\widehat{u}_{1}, \psi\right\rangle}{\left\langle 1-G_{2} L_{10}^{-1} u_{0}, \psi^{2}\right\rangle},
$$

which is a real constant. Hence a real localized solution for $u_{1}$ has been obtained.

With the above $b_{1}$ value, the solvability condition of the $u_{2}$ equation (4.31) is satisfied. Thus this equation admits a real localized particular solution $\widehat{u}_{2}$, and its general solution is

$$
u_{2}=\widehat{u}_{2}+b_{2} \psi
$$

where $b_{2}$ is another constant to be determined.

Inserting this $u_{2}$ solution into the $u_{3}$ equation (4.27), this equation becomes

$$
L_{10} u_{3}=b_{2} \psi\left(1-G_{2} u_{1}\right)+\widehat{u}_{2}\left(1-G_{2} u_{1}\right)-\frac{1}{3 !} G_{3} u_{1}^{3} .
$$


By Lemma 1, the solvability condition of this $u_{3}$ equation is that its right hand side be orthogonal to $\psi$. Utilizing the $u_{1}$ solution (4.29) and the conditions of Case 2, we see that

$$
\left\langle\psi\left(1-G_{2} u_{1}\right), \psi\right\rangle=\left\langle 1-G_{2} L_{10}^{-1} u_{0}, \psi^{2}\right\rangle \neq 0 .
$$

Thus the solvability condition of Eq. (4.35) yields a unique real $b_{2}$ value,

$$
b_{2}=-\frac{\left\langle\widehat{u}_{2}\left(1-G_{2} u_{1}\right)-G_{3} u_{1}^{3} / 3 !, \psi\right\rangle}{\left\langle\psi\left(1-G_{2} u_{1}\right), \psi\right\rangle},
$$

hence a real localized $u_{2}$ solution (4.34) is then obtained. At this $b_{2}$ value, Eq. (4.35) admits a real localized particular solution $\widehat{u}_{3}$, and its general solution is

$$
u_{3}=\widehat{u}_{3}+b_{3} \psi
$$

where $b_{3}$ is another constant to be determined.

Pursuing this calculation to higher orders, it is easy to see that for any $n \geq 2$, the $u_{n}$ solution is of the form

$$
u_{n}=\widehat{u}_{n}+b_{n} \psi,
$$

where $\widehat{u}_{n}$ is a real localized particular solution of the $u_{n}$ equation, and $b_{n}$ is a constant to be determined from the solvability condition of the $u_{n+1}$ equation. The $u_{n+1}$ equation is always of the form

$$
L_{10} u_{n+1}=\left(1-G_{2} u_{1}\right) u_{n}+\mathcal{F}_{n+1}\left(u_{0}, u_{1}, \ldots, u_{n-1} ; \mathbf{x}\right),
$$

where $\mathcal{F}_{n+1}$ is some real function which depends on the already-obtained real localized solutions $u_{0}, u_{1}, \ldots, u_{n-1}$ as well as $\mathbf{x}$. Inserting the $u_{n}$ solution (4.38) into (4.39) and utilizing Eq. (4.36), the solvability condition of (4.39) is met at a unique real $b_{n}$ value, hence a real localized $u_{n}$ solution (4.38) is obtained. Meanwhile, since the solvability condition of (4.39) is met, a real localized particular solution $\widehat{u}_{n+1}$ exists, and the general $u_{n+1}$ solution is of the form (4.38) with the index $n$ replaced by $n+1$. This process then repeats itself. Hence a real-valued and localized perturbation series solution (4.23) is constructed to all orders, and it gives a branch of real-valued solitary waves $u^{0}(\mathbf{x} ; \mu)$ which exists on both sides of $\mu=\mu_{0}$ and depends smoothly on $\mu$.

(ii) Next we construct two additional solitary wave branches which exist on only one side of $\mu=\mu_{0}$ and merge with the above smooth solution branch at $\mu=\mu_{0}$. These additional solitary wave branches have the following perturbation series expansion

$$
u(\mathbf{x} ; \mu)=\sum_{k=0}^{\infty}\left(\mu-\mu_{0}\right)^{k / 2} u_{k}(\mathbf{x}) .
$$

This perturbation series is of the same form as (4.3) in Case 1. Thus when this perturbation series is substituted into Eq. (2.3), the resulting equations for $u_{k}$ are the same as (4.4)-(4.8) before. But since the conditions for Case 2 are different from those for Case 1, solutions $u_{k}$ for the perturbation series here will differ from those in (4.3), as we will show below.

First, the equation (4.4) for $u_{0}$ is satisfied automatically since $u_{0}$ is a solitary wave of (2.3) at $\mu=\mu_{0}$. The solution $u_{1}$ to Eq. (4.5), under Assumption 1, is

$$
u_{1}=b_{1} \psi
$$


where $b_{1}$ is a constant to be determined. Inserting this $u_{1}$ solution into the $u_{2}$ equation (4.6), we get

$$
L_{10} u_{2}=u_{0}-\frac{1}{2} b_{1}^{2} G_{2} \psi^{2} .
$$

Due to conditions of Case 2, both $u_{0}$ and $G_{2} \psi^{2}$ are orthogonal to $\psi$. Thus by Lemma 1, both $L_{10}^{-1} u_{0}$ and $L_{10}^{-1}\left(G_{2} \psi^{2}\right)$ exist and are certain real localized functions. Hence the solution $u_{2}$ to Eq. (4.42) is

$$
u_{2}=L_{10}^{-1} u_{0}-\frac{1}{2} b_{1}^{2} L_{10}^{-1}\left(G_{2} \psi^{2}\right)+b_{2} \psi,
$$

where $b_{2}$ is another constant to be determined. Inserting these $u_{1}$ and $u_{2}$ solutions into (4.7), the $u_{3}$ equation is

$$
L_{10} u_{3}=b_{1}\left\{\left(1-G_{2} L_{10}^{-1} u_{0}\right) \psi-\frac{1}{3 !} b_{1}^{2}\left[G_{3} \psi^{3}-3 G_{2} \psi L_{10}^{-1}\left(G_{2} \psi^{2}\right)\right]-b_{2} G_{2} \psi^{2}\right\} .
$$

In view of the conditions of Case 2 , the solvability condition of this $u_{3}$ equation yields the $b_{1}$ value as

$$
b_{1}= \pm \nu, \quad \nu \equiv \sqrt{\frac{6\left\langle 1-G_{2} L_{10}^{-1} u_{0}, \psi^{2}\right\rangle}{\left\langle G_{3}, \psi^{4}\right\rangle-3\left\langle G_{2} \psi^{2}, L_{10}^{-1}\left(G_{2} \psi^{2}\right)\right\rangle}} .
$$

Two $b_{1}$ values $\pm \nu$ are obtained which are opposite of each other. Inserting the corresponding $u_{1}$ solutions (4.41) into (4.40), we then get two solutions $u^{ \pm}(\mathbf{x} ; \mu)$ as perturbation series

$$
u^{ \pm}(\mathbf{x} ; \mu)=u_{0}(\mathbf{x}) \pm \nu\left(\mu-\mu_{0}\right)^{1 / 2} \psi(\mathbf{x})+O\left(\mu-\mu_{0}\right)
$$

where $\nu$ is given in (4.45). If the numerator and denominator under the square root of (4.45) have the same sign, then $\nu$ is real. In this case, two real localized perturbation series solutions (4.46) are obtained when $\mu>\mu_{0}$. If the numerator and denominator in (4.45) have the opposite sign, then $\nu$ is purely imaginary. In this case, two real localized perturbation series solutions (4.46) are obtained when $\mu<\mu_{0}$.

Next we show that the two real localized perturbation series solutions (4.46), which exist on only one side of $\mu=\mu_{0}$, can be constructed to all orders of $\left(\mu-\mu_{0}\right)^{1 / 2}$. With the choice of $b_{1}$ values in (4.45), the solvability condition of the $u_{3}$ equation (4.44) is met, thus the $u_{3}$ solution is

$$
u_{3}=b_{1}\left[\widehat{u}_{3}-b_{2} L_{10}^{-1}\left(G_{2} \psi^{2}\right)+b_{3} \psi\right]
$$

where $\widehat{u}_{3}$ is a real localized function which satisfies the equation

$$
L_{10} \widehat{u}_{3}=\left(1-G_{2} L_{10}^{-1} u_{0}\right) \psi-\frac{1}{3 !} \nu^{2}\left[G_{3} \psi^{3}-3 G_{2} \psi L_{10}^{-1}\left(G_{2} \psi^{2}\right)\right]
$$

and $b_{3}$ is a constant to be determined. Inserting these $u_{1}, u_{2}$ and $u_{3}$ solutions into (4.8), the $u_{4}$ equation becomes

$$
\begin{aligned}
L_{10} u_{4}= & b_{2}\left\{\left(1-G_{2} L_{10}^{-1} u_{0}\right) \psi-\frac{1}{2} b_{1}^{2}\left[G_{3} \psi^{3}-3 G_{2} \psi L_{10}^{-1}\left(G_{2} \psi^{2}\right)\right]\right\} \\
& -\frac{1}{2} b_{2}^{2} G_{2} \psi^{2}+\mathcal{F}_{4}\left(u_{0}, \psi, b_{1}^{2}, \mathbf{x}\right)-b_{1}^{2} b_{3} G_{2} \psi^{2}
\end{aligned}
$$


where $\mathcal{F}_{4}$ is a real localized function which depends on $u_{0}, \psi, b_{1}^{2}$ and other already-obtained real functions (such as $\widehat{u}_{3}$ ). Utilizing the $b_{1}$ formula (4.45) as well as conditions of Case 2, the solvability condition of this $u_{4}$ equation is met at the unique real $b_{2}$ value,

$$
b_{2}=\frac{\left\langle\mathcal{F}_{4}, \psi\right\rangle}{2\left\langle 1-G_{2} L_{10}^{-1} u_{0}, \psi^{2}\right\rangle} .
$$

When this $b_{2}$ value is inserted into (4.43), a real localized $u_{2}$ solution is then obtained. Notice that this $b_{2}$ is the same for both choices $\pm \nu$ of $b_{1}$ in the $u_{1}$ solution (4.41), thus $u_{2}(\mathbf{x})$ is the same for both branches of the perturbation series solutions $u^{ \pm}(\mathbf{x} ; \mu)$ in (4.46).

For the $b_{2}$ value given in (4.50), the solvability condition of the $u_{4}$ equation (4.49) is satisfied, thus this equation admits the following localized solution

$$
u_{4}=\widehat{u}_{4}-\nu^{2} b_{3} L_{10}^{-1}\left(G_{2} \psi^{2}\right)+b_{4} \psi
$$

where $\widehat{u}_{4}$ is a real localized function which satisfies the $u_{4}$ equation (4.49) but without the last $\left(b_{3}\right)$ term, and $b_{4}$ is a new constant to be determined.

Starting from $n \geq 5$, the $u_{n}$ equation can be derived from (2.3) and the expansion (4.40), and is all of the form

$$
\begin{gathered}
L_{10} u_{2 n+1}=u_{2 n-1}-G_{2}\left(u_{1} u_{2 n}+u_{2} u_{2 n-1}\right)-\frac{1}{2} G_{3} u_{1}^{2} u_{2 n-1}+b_{1} \mathcal{H}_{2 n+1}\left(u_{0}, \psi, b_{1}^{2}, \mathbf{x}\right), \quad n \geq 2, \\
L_{10} u_{2 n+2}=u_{2 n}-G_{2}\left(u_{1} u_{2 n+1}+u_{2} u_{2 n}\right)-\frac{1}{2} G_{3} u_{1}^{2} u_{2 n}+\mathcal{H}_{2 n+2}\left(u_{0}, \psi, b_{1}^{2}, \mathbf{x}\right), \quad n \geq 2,
\end{gathered}
$$

where $\mathcal{H}_{2 n+1}$ and $\mathcal{H}_{2 n+2}$ are real localized functions which depend on $u_{0}, \psi, b_{1}^{2}$ and other already fully determined real quantities (such as $\widehat{u}_{3}, \widehat{u}_{4}, b_{2}$, etc). Using the method of induction as well as conditions of Case 2, we can show that all $u_{n}$ solutions are of the form

$$
\begin{array}{ll}
u_{2 n+1}=b_{1}\left[\widehat{u}_{2 n+1}-b_{2 n} L_{10}^{-1}\left(G_{2} \psi^{2}\right)+b_{2 n+1} \psi\right], & n \geq 1, \\
u_{2 n+2}=\widehat{u}_{2 n+2}-\nu^{2} b_{2 n+1} L_{10}^{-1}\left(G_{2} \psi^{2}\right)+b_{2 n+2} \psi, & n \geq 1,
\end{array}
$$

where $\widehat{u}_{2 n+1}$ and $\widehat{u}_{2 n+2}$ are certain real localized functions, and $b_{2 n+1}, b_{2 n+2}$ are real constants which are determined uniquely from the solvability conditions of the $u_{2 n+3}$ and $u_{2 n+4}$ equations. We can also show that $\widehat{u}_{2 n+1}, \widehat{u}_{2 n+2}, b_{2 n+1}$ and $b_{2 n+2}$ depend on $b_{1}^{2}$ as a whole and are thus the same for both solution branches $u^{ \pm}(\mathbf{x} ; \mu)$. Inserting these solutions into the perturbation series (4.40), we obtain two branches of solitary waves $u^{ \pm}(\mathbf{x} ; \mu)$ whose perturbation series expansions are

$$
\begin{aligned}
u^{ \pm}(\mathbf{x} ; \mu)= & u_{0}(\mathbf{x})+\sum_{n=1}^{\infty}\left(\mu-\mu_{0}\right)^{n} u_{2 n}(\mathbf{x}) \\
& \pm \nu\left(\mu-\mu_{0}\right)^{1 / 2}\left\{\psi(\mathbf{x})+\sum_{n=1}^{\infty}\left(\mu-\mu_{0}\right)^{n} \widetilde{u}_{2 n+1}(\mathbf{x})\right\},
\end{aligned}
$$

where real localized functions $u_{2 n}$ are given by (4.43) and (4.55), real localized functions $\widetilde{u}_{2 n+1}$ are as $u_{2 n+1}$ in (4.54) but without the $b_{1}$ factor, and $\nu$ is given in (4.45). These two real solitary waves exist on the side of $\mu>\mu_{0}\left(\mu<\mu_{0}\right)$ when $\nu$ is real (purely imaginary). When $\mu \rightarrow \mu_{0}$, they both 
approach $u_{0}(\mathbf{x})$, thus these $u^{ \pm}(\mathbf{x} ; \mu)$ solution branches merge with the smooth $u^{0}(\mathbf{x} ; \mu)$ solution branch in (4.23) at $\mu=\mu_{0}$.

The existence of the smooth solution branch $u^{0}(\mathbf{x} ; \mu)$ in (4.23) on both sides of $\mu=\mu_{0}$ as well as two additional solution branches $u^{ \pm}(\mathbf{x} ; \mu)$ in (4.56) on only one side of $\mu=\mu_{0}$ indicates that a pitchfork bifurcation occurs at $\mu=\mu_{0}$.

\section{Case 3: transcritical bifurcations}

Now we consider the third case of Theorem 1. We will show that under conditions of this case, two smooth branches of solitary waves exist on both sides of $\mu=\mu_{0}$, and these branches intersect at $\mu=\mu_{0}$ where solitary waves on the two branches become identical. Thus a transcritical bifurcation occurs at $\mu=\mu_{0}$.

In this third case, we seek solitary wave solutions which exist on both sides of $\mu=\mu_{0}$ and depend smoothly on $\mu$ near $\mu=\mu_{0}$. The perturbation series expansion of such solutions is

$$
u(\mathbf{x} ; \mu)=\sum_{k=0}^{\infty}\left(\mu-\mu_{0}\right)^{k} u_{k}(\mathbf{x}) .
$$

The form of this expansion is the same as (4.23) in Case 2, thus the equations for $u_{k}$ are also the same as (4.24)-(4.28) before. However, the solutions to these equations will differ from the previous ones in Case 2 due to different conditions of the present case.

The $u_{0}$ equation (4.24) is satisfied automatically since $u_{0}$ is a solitary wave of Eq. (2.3) at $\mu=\mu_{0}$. Under conditions of Case 3 , the solvability condition of the $u_{1}$ equation (4.25), $\left\langle u_{0}, \psi\right\rangle=0$, is met. Thus by Lemma 1 , localized $u_{1}$ solutions of the form

$$
u_{1}=L_{10}^{-1} u_{0}+b_{1} \psi
$$

are admitted. Here $L_{10}^{-1} u_{0}$ is a real and localized particular solution to Eq. (4.25), and $b_{1}$ is a constant to be determined. Inserting this $u_{1}$ solution into the $u_{2}$ equation (4.26), this equation becomes

$$
L_{10} u_{2}=L_{10}^{-1} u_{0}-\frac{1}{2} G_{2}\left(L_{10}^{-1} u_{0}\right)^{2}+b_{1}\left(1-G_{2} L_{10}^{-1} u_{0}\right) \psi-\frac{1}{2} b_{1}^{2} G_{2} \psi^{2} .
$$

The solvability condition of this equation gives the following quadratic equation for $b_{1}$ :

$$
\left\langle G_{2}, \psi^{3}\right\rangle b_{1}^{2}-2\left\langle 1-G_{2} L_{10}^{-1} u_{0}, \psi^{2}\right\rangle b_{1}+\left\langle G_{2}\left(L_{10}^{-1} u_{0}\right)^{2}-2 L_{10}^{-1} u_{0}, \psi\right\rangle=0 .
$$

Under conditions of Case 3, the coefficient of the $b_{1}^{2}$ term in this quadratic equation is nonzero, and

$$
\Delta \equiv\left\langle 1-G_{2} L_{10}^{-1} u_{0},, \psi^{2}\right\rangle^{2}-\left\langle G_{2}, \psi^{3}\right\rangle\left\langle G_{2}\left(L_{10}^{-1} u_{0}\right)^{2}-2 L_{10}^{-1} u_{0}, \psi\right\rangle>0 .
$$

Thus this quadratic equation admits the following two real roots

$$
b_{1}=b_{1}^{ \pm} \equiv \frac{\left\langle 1-G_{2} L_{10}^{-1} u_{0}, \psi^{2}\right\rangle \pm \sqrt{\Delta}}{\left\langle G_{2}, \psi^{3}\right\rangle} .
$$

For each of these two $b_{1}$ values, a real localized $u_{1}$ solution (4.58) is obtained. In addition, a real and localized particular solution $\widehat{u}_{2}$ to the $u_{2}$ equation (4.59) exists, hence the $u_{2}$ solution is

$$
u_{2}=\widehat{u}_{2}+b_{2} \psi
$$


where $b_{2}$ is a new constant to be determined.

Inserting the above $u_{2}$ solution into the $u_{3}$ equation (4.27), we get

$$
L_{10} u_{3}=b_{2}\left(1-G_{2} u_{1}\right) \psi+\left(1-G_{2} u_{1}\right) \widehat{u}_{2}-\frac{1}{3 !} G_{3} u_{1}^{3} .
$$

Utilizing the $u_{1}$ solution (4.58) and the $b_{1}$ formula (4.61), we find that

$$
\left\langle\left(1-G_{2} u_{1}\right) \psi, \psi\right\rangle=\left\langle 1-G_{2} L_{10}^{-1} u_{0}, \psi^{2}\right\rangle-b_{1}\left\langle G_{2}, \psi^{3}\right\rangle=\mp \sqrt{\Delta} \neq 0 .
$$

Thus the solvability condition of Eq. (4.63) yields a real constant $b_{2}$ as

$$
b_{2}=-\frac{\left\langle\left(1-G_{2} u_{1}\right) \widehat{u}_{2}-G_{3} u_{1}^{3} / 3 !, \psi\right\rangle}{\left\langle\left(1-G_{2} u_{1}\right) \psi, \psi\right\rangle} .
$$

For this $b_{2}$ value, the solvability condition of the $u_{3}$ equation (4.63) is satisfied, thus this equaiton admits a real localized particular solution $\widehat{u}_{3}$, and the general $u_{3}$ solution is

$$
u_{3}=\widehat{u}_{3}+b_{3} \psi
$$

where $b_{3}$ is another constant to be determined.

Pursuing this calculation to higher orders, it is easy to see that for any $n \geq 2$, the $u_{n}$ solution is of the form

$$
u_{n}=\widehat{u}_{n}+b_{n} \psi,
$$

where $\widehat{u}_{n}$ is a real localized particular solution of the $u_{n}$ equation, and $b_{n}$ is a real constant to be determined from the solvability condition of the $u_{n+1}$ equation. The $u_{n+1}$ equation is always of the form

$$
L_{10} u_{n+1}=\left(1-G_{2} u_{1}\right) u_{n}+\mathcal{F}_{n+1}\left(u_{0}, u_{1}, \ldots, u_{n-1} ; \mathbf{x}\right),
$$

where $\mathcal{F}_{n+1}$ is some real function which depends on the already-obtained real localized solutions $u_{0}, u_{1}, \ldots, u_{n-1}$ and $\mathbf{x}$. Inserting the $u_{n}$ solution (4.66) into (4.67) and in view of Eq. (4.64), the solvability condition of (4.67) then yields a unique real value for the constant $b_{n}$.

In the above solution process, since $b_{1}$ can take either one of the two real roots $b_{1}^{ \pm}$in (4.61), $u_{1}$ in (4.58) then has two corresponding solutions $u_{1}^{ \pm}$. These two $u_{1}$ solutions cascade up to higher orders, and thus all $u_{n}$ functions have two solutions $u_{n}^{ \pm}$. Consequently, two real-valued and localized perturbation series solutions

$$
u^{ \pm}(\mathbf{x} ; \mu)=u_{0}(\mathbf{x})+\sum_{k=1}^{\infty}\left(\mu-\mu_{0}\right)^{k} u_{k}^{ \pm}(\mathbf{x})
$$

are obtained to all orders, and they provide two branches of real-valued solitary waves $u^{ \pm}(\mathbf{x} ; \mu)$ which exist on both sides of $\mu=\mu_{0}$ and depend smoothly on $\mu$. When $\mu \rightarrow \mu_{0}$, both $u^{ \pm}(\mathbf{x} ; \mu)$ approach $u_{0}(\mathbf{x})$, thus these two solution branches intersect at $\mu=\mu_{0}$. As a result, a transcritical bifurcation occurs at $\mu=\mu_{0}$. This completes the proof of Theorem 1 .

Next, we prove Theorem 2 on power diagrams near bifurcation points. 
Proof of Theorem 2 The power formula (3.4) of saddle-node bifurcations can be derived easily from the perturbation series solutions (4.3) and the $u_{1}$ solution (4.9) with $b_{1}$ given by Eq. (4.11). The power formula (3.6) for the smooth solution branch $u^{0}(\mathbf{x} ; \mu)$ in a pitchfork bifurcation can be derived readily from the perturbation series solutions (4.23) and the $u_{1}$ solution (4.29). To derive the power formula (3.8) for the two bifurcated solution branches in a pitchfork bifurcation, we substitute the $u_{1}, u_{2}$ solutions in (4.41) and (4.43) into the expansion (4.56), and find that the power function is given by (3.8), where

$$
P_{1}=2\left\langle u_{0}, L_{10}^{-1} u_{0}\right\rangle+b_{1}^{2}\left[\langle\psi, \psi\rangle-\left\langle u_{0}, L_{10}^{-1}\left(G_{2} \psi^{2}\right)\right\rangle\right]
$$

whose value is the same for both bifurcated branches. Since $L_{10}^{-1}$ is self-adjoint and $L_{10}^{-1} u_{0}$ exists (by Lemma 1), this $P_{1}$ coefficient can then be rewritten as (3.9) (here the $b_{1}$ formula (4.45) is also used). The power formula (3.10) for transcritical bifurcations can be derived easily from the perturbation series solutions (4.68) and the $u_{1}, u_{2}$ solutions (4.58), (4.62).

\section{Numerical examples of solitary wave bifurcations}

In this section, we present numerical examples for these three types of solitary wave bifurcations, and compare them with the analytical results presented in Theorems 1 and 2. So far, examples of saddle-node and pitchfork bifurcations of solitary waves have been reported in the GNLS equations (2.1) with various potentials and nonlinearities [2, 8, 9, 11, 13, 14, 15, 16, 17, 18, 19]. Here we will present some new examples of saddle-node and pitchfork bifurcations in the GNLS equations which exhibit interesting novel features. In addition, we will present the first example of transcritical bifurcation in these GNLS equations.

\section{Example 1: combined saddle-node and double-pitchfork bifurcations}

The first example we choose is the one-dimensional GNLS equation (2.1) with a symmetric double-well potential and cubic-quintic nonlinearity:

$$
i U_{t}+U_{x x}-V(x) U+|U|^{2} U-\gamma|U|^{4} U=0,
$$

where the symmetric double-well potential $V(x)$ is taken of the form

$$
V(x)=-V_{0}\left[\operatorname{sech}^{2}\left(x+x_{0}\right)+\operatorname{sech}^{2}\left(x-x_{0}\right)\right],
$$

$V_{0}>0$ is the potential depth, $2 x_{0}$ is the separation between the two wells, and $\gamma>0$ is the coefficient of the quintic nonlinearity. Notice that the cubic and quintic nonlinear terms in (5.1) have the opposite sign, and the quintic term induces a self-defocusing effect which counters the self-focusing effect of the cubic term. One may also view this opposing cubic-quintic nonlinearity as a Taylor-series approximation to the saturable nonlinearity in photorefractive crystals [26]. The parameter values in the above GNLS model are chosen as

$$
V_{0}=2.8, \quad x_{0}=1.5, \quad \gamma=0.25
$$

Solitary waves in Eq. (5.1) are sought of the form (2.2), where $u(x)$ is a real localized function satisfying the equation

$$
u_{x x}-\mu u-V(x) u+u^{3}-\gamma u^{5}=0 .
$$


When $u(x)$ is infinitesimal, the linear Schrödinger operator of Eq. (5.4) admits a positive symmetric discrete eigenfunction at eigenvalue $\mu \approx 1.7896$. This eigenmode is the ground state of the underlying double-well potential. From this linear (infinitesimal) eigenmode, a family of positive symmetric solitary waves bifurcates out. The power curve of this symmetric-soliton family is shown in Fig. 2 (blue curve in the upper left panel). We have computed the spectra of the linearization operator $L_{1}$ for these solitary waves, and found that their spectra contain a simple zero eigenvalue at three locations marked by letters 'A,B,C' on the power curve. This is evidenced in the upper right panel of Fig. 2, where the $L_{1}$-spectra of solitary waves at these three locations are displayed. Notice that at locations 'A,B', the second largest eigenvalue of the spectrum is zero, while at location 'C', the largest eigenvalue is zero. At these three locations, solitary waves $u_{0}(x)$ and eigenfunctions $\psi(x)$ of the zero eigenvalue in $L_{1}$ 's spectra are plotted in the lower row of Fig. 2 (as solid blue and dashed red curves respectively). Notice that eigenfunctions $\psi(x)$ at points 'A,B' are anti-symmetric, while the eigenfunction at point ' $\mathrm{C}$ ' is symmetric.
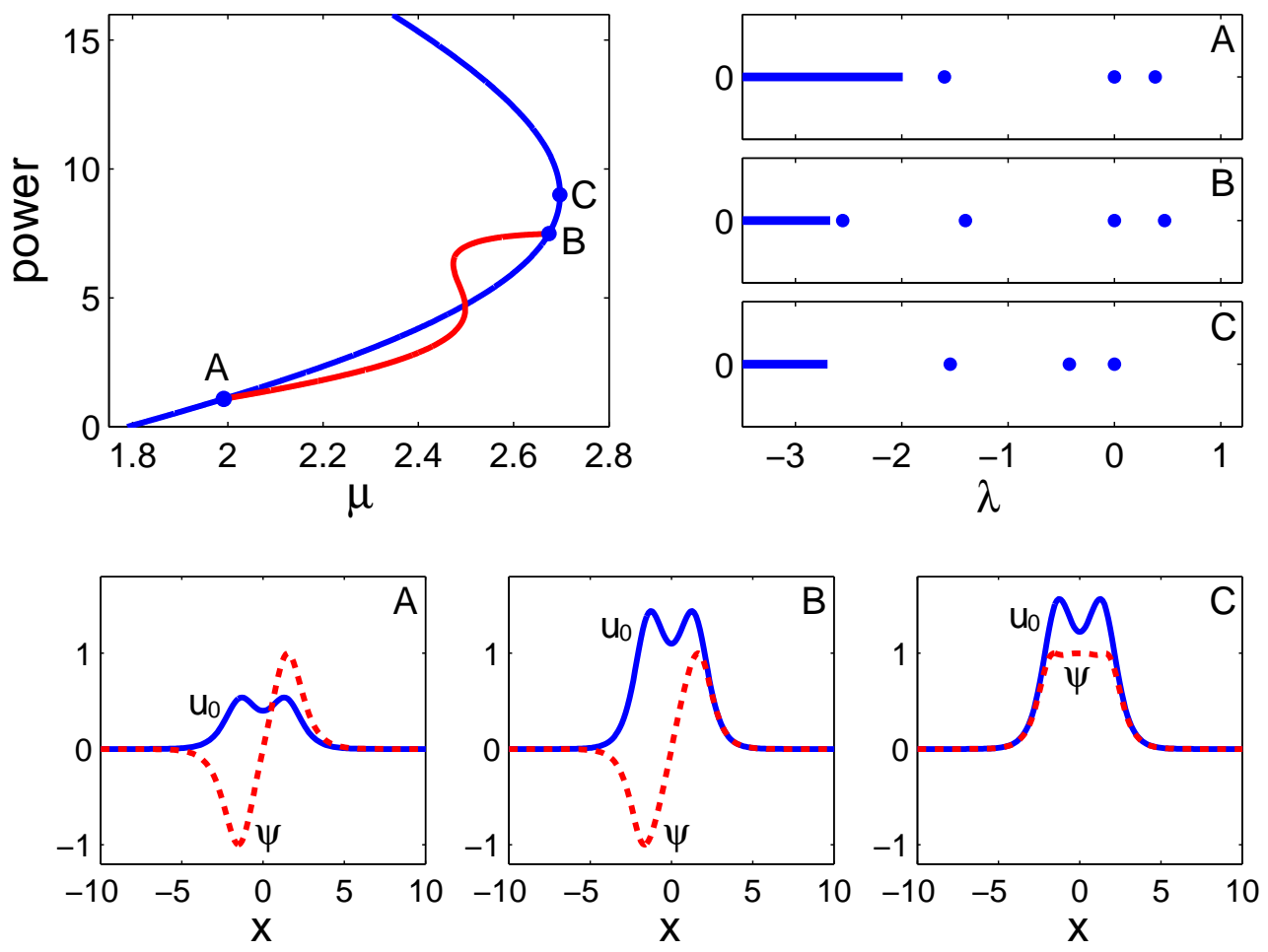

Figure 2: Bifurcations of solitary waves in Example 1. Upper left: the power diagram; the blue curve is for the family of symmetric solitary waves, and the red curve is for the family of asymmetric solitary waves which bifurcate out from points 'A, B' through double pitchfork bifurcations. Upper right: $L_{1}$ 's spectra for solitary waves at bifurcation points 'A,B,C' of the power diagram. Lower row: solitary waves $u_{0}(x)$ and eigenfunctions $\psi(x)$ of $L_{1}$ 's zero eigenvalue at bifurcation points 'A,B,C' (the eigenfunctions are normalized to have unit amplitude).

At these 'A,B,C' points, zero is a simple discrete eigenvalue of $L_{1}$, thus Assumption 1 is met and Theorem 1 applies. In addition, for the present example,

$$
G_{2}=6 u_{0}-20 \gamma u_{0}^{3}, \quad G_{3}=6-60 \gamma u_{0}^{2}
$$


Now we use our analytical criterion in Theorem 1 to determine if and what bifurcations occur at these points.

At points 'A,B', it is easy to see from symmetry that

$$
\left\langle u_{0}, \psi\right\rangle=\left\langle G_{2}, \psi^{3}\right\rangle=0
$$

In addition, when the eigenfunction $\psi$ is normalized to have unit amplitude (see Fig. 2, lower row), we find numerically that at point ' $\mathrm{A}$ ',

$$
\left\langle 1-G_{2} L_{10}^{-1} u_{0}, \psi^{2}\right\rangle=-4.9313, \quad\left\langle G_{3}, \psi^{4}\right\rangle-3\left\langle G_{2} \psi^{2}, L_{10}^{-1}\left(G_{2} \psi^{2}\right)\right\rangle=-58.4035
$$

and at point 'B',

$$
\left\langle 1-G_{2} L_{10}^{-1} u_{0}, \psi^{2}\right\rangle=23.9913, \quad\left\langle G_{3}, \psi^{4}\right\rangle-3\left\langle G_{2} \psi^{2}, L_{10}^{-1}\left(G_{2} \psi^{2}\right)\right\rangle=-110.9244 .
$$

Then according to Theorem 1, pitchfork bifurcations occur at both ' $\mathrm{A}$ ' and ' $\mathrm{B}$ ' points. In addition, the new (asymmetric) solitary waves bifurcate out on the right side of point 'A' and on the left side of point ' $\mathrm{B}$ '.

At point ' $\mathrm{C}$ ', we find that

$$
\left\langle u_{0}, \psi\right\rangle=6.4879, \quad\left\langle G_{2}, \psi^{3}\right\rangle=-21.0632
$$

thus according to Theorem 1, a saddle-node bifurcation occurs at this point. In addition, the bifurcated solutions appear on the left side of point ' $\mathrm{C}$ '.

These analytical predictions of bifurcations prove to be completely correct. Specifically, at points 'A,B', symmetry-breaking pitchfork bifurcations occur. The two bifurcated asymmetric solitary waves $u^{ \pm}(x ; \mu)$ are related to each other by a mirror reflection in $x$, i.e., $u^{+}(-x ; \mu)=u^{-}(x ; \mu)$, and their power curves (which are identical) are displayed as the red line in Fig. 2 (upper left panel). Notice that these bifurcated solutions appear on the right side of point 'A' and on the left side of point ' $\mathrm{B}$ ', as predicted by the above analysis. To illustrate solution profiles before and after these bifurcations, we focus on point ' $\mathrm{A}$ '. The power diagram near this bifurcation point is amplified from that in Fig. 2 and shown in Fig. 3 (first panel from the left). Notice that the power curves near this bifurcation point are linear functions of $\mu$, in agreement with Theorem 2 and Fig. 1(b). We have also compared the slopes of the power curves at point 'A' in Fig. 3 with the analytical power slopes in Eqs. (3.6)-(3.9), and found excellent agreement. At three locations 'a,b,c' on the two sides of the bifurcation point 'A' in the power diagram, profiles of the solitary waves are displayed in Fig. 3(a-c) respectively. Solutions in Fig. 3(a,b) are symmetric and lie on the symmetric branch of the power diagram (blue line), while the two solutions in Fig. 3(c) are asymmetric and lie on the asymmetric (bifurcated) branch of the power diagram (red line). Notice that on the left side of the bifurcation point, there is a single (symmetric) solitary wave (see Fig. 3(a)); but on the right side of the bifurcation point, there are three solitary waves, one symmetric (see Fig. 3(b)) and the other two asymmetric (see Fig. 3(c)). These behaviors of the pitchfork bifurcation agree fully with our analytical results as well as the schematic plots in Fig. 1(b).

It is interesting to observe from Fig. 2 (upper left panel) that the asymmetric soliton branch starts out from point ' $\mathrm{A}$ ' and terminates at point ' $\mathrm{B}$ ', thus it appears and then disappears through 

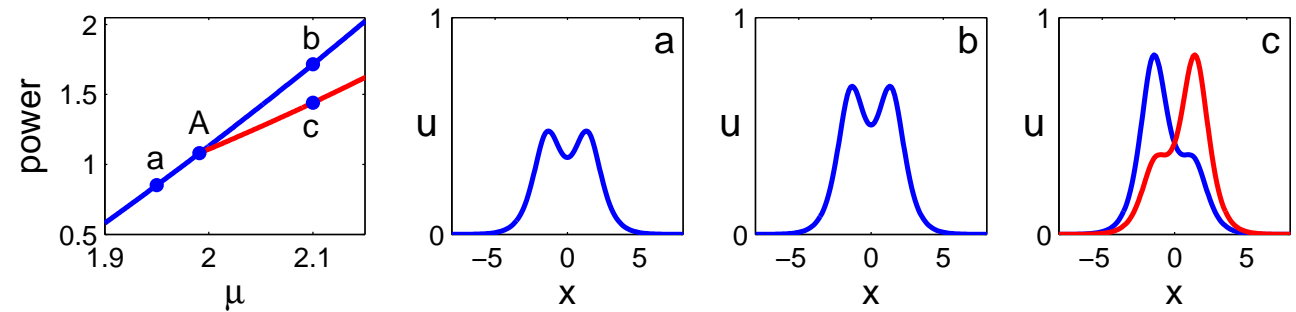

Figure 3: Power diagram and profiles of solitary waves near the pitchfork bifurcation point 'A' in Fig. 2. First panel: power diagram; $(\mathrm{a}, \mathrm{b}, \mathrm{c})$ solitary waves at locations marked by the same letters on the power diagram.

double pitchfork bifurcations. In between, its power curve exhibits a ' $S$ ' shape, indicating that double saddle-node bifurcations also occur on this asymmetric branch. These features of bifurcations are quite novel for the GNLS equations (2.1). In a different nonlinear wave system, namely nonlinear saturable couplers, a similar double pitchfork bifurcation also exists [12].

At point ' $\mathrm{C}$ ' of Fig. 2, we have found that a saddle-node bifurcation occurs as predicted. This is already obvious from the power diagram in Fig. 2, which shows that the power curve turns around at this point. The power diagram near this saddle-node bifurcation point ' $\mathrm{C}$ ' is amplified and shown again in Fig. 4. This numerical power curve is compared with the analytical saddle-node power formula (3.4) and complete agreement is obtained. At two locations 'a,b' of the power curve below and above the bifurcation, profiles of the solitary waves are displayed in Fig. 4(a,b). These solutions are all symmetric, and their amplitudes vary while going through the bifurcation.
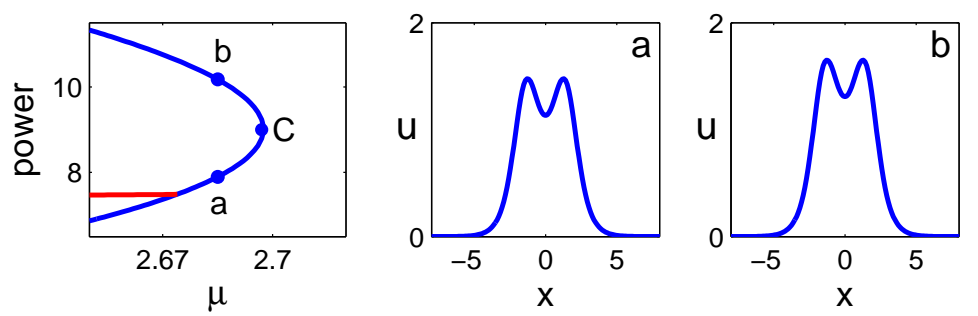

Figure 4: Power diagram and profiles of solitary waves near the saddle-node bifurcation point 'C' in Fig. 2. Left panel: power diagram; $(a, b)$ solitary waves at locations marked by the same letters on the power diagram.

\section{Example 2: power loop phenomena}

Our second example is still the GNLS equation with opposing cubic and quintic nonlinearities,

$$
i U_{t}+U_{x x}-V(x) U+|U|^{2} U-0.15|U|^{4} U=0
$$

but the double-well potential $V(x)$ is now asymmetric instead:

$$
V(x)=-3.5 \operatorname{sech}^{2}(x+1.5)-3 \operatorname{sech}^{2}(x-1.5) .
$$

This potential is displayed in Fig. 5(a). As usual, solitary waves in this equation are sought of the form (2.2), where $u(x)$ is a real localized function. We find that in this system, there exist a family 
of positive solitary waves whose power curve forms a closed loop. This power loop is displayed in Fig. 5(b). This power loop shows that this family of solitary waves has a non-zero minimal power and a finite maximal power, and it exists over a finite propagation-constant interval. In addition, four saddle-node bifurcations are clearly visible on this loop. We have checked that at these saddlenode bifurcation points, the bifurcation conditions in Theorem 1 (Case 1) are all satisfied. At four locations of the power loop, three of them ('c,e,f') being saddle-node bifurcation points and the remaining one (' $d$ ') slightly below a saddle-node bifurcation point, profiles of the solitary waves are displayed in Fig. 5(c-f). It is seen that the energy of these solitary waves resides primarily in the shallower (right) well of the potential. Thus this family of solitary waves is different from the family of ground-state solitary waves in this system, whose energy resides primarily in the deeper (left) well of the potential. One may notice that this power loop in Fig. 5)(b) self-crosses itself in the middle (above point ' $d$ '). This power-curve crossing does not signal a transcritical bifurcation however, because as $\mu$ approaches this crossing point along the two intersecting curves, the solitary waves do not approach each other. This power loop phenomenon of solitary waves has not been reported before in the GNLS equations (2.1) (to the author's knowledge), but a similar momentum loop phenomenon for solitons sitting on constant backgrounds has been discovered in the externally driven NLS equation [27].
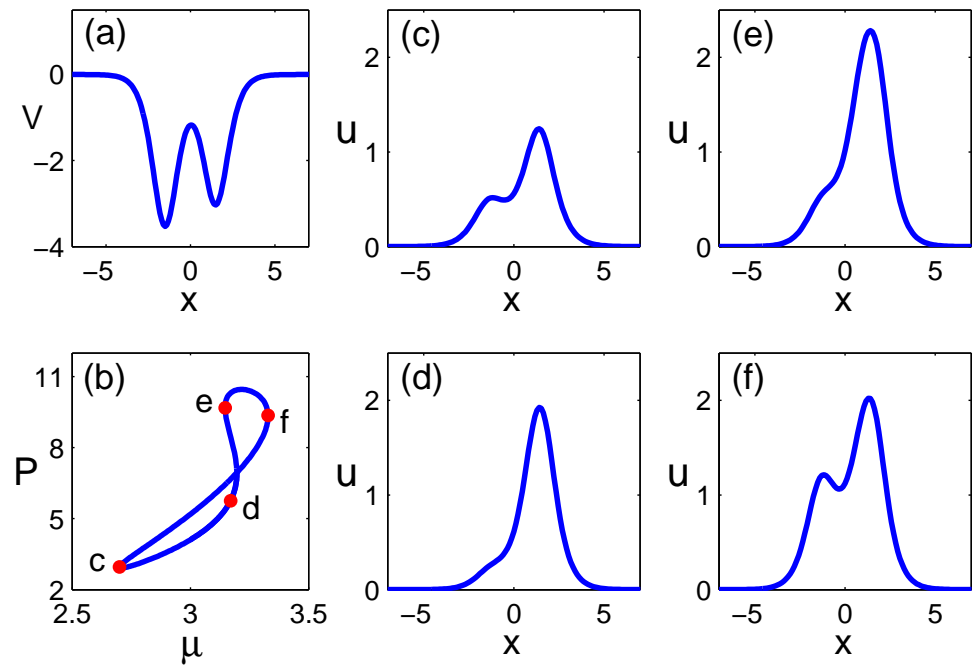

Figure 5: Power-loop phenomenon in Example 2 (i.e., Eq. (5.5)). (a) The asymmetric double-well potential $V(x)$ in Eq. (5.6); (b) the power loop; (c-f) profiles of solitary waves at locations marked by the same letters on the power loop of (b).

\section{Example 3: transcritical bifurcation}

Our last example is the GNLS equation with competing cubic, quintic and seventh-power nonlinearities,

$$
i U_{t}+U_{x x}-V(x) U+|U|^{2} U-0.2|U|^{4} U+\kappa|U|^{6} U=0,
$$

where $V(x)$ is the same asymmetric double-well potential (5.6) as in Example 2, and $\kappa$ is a real constant. In this example, a transcritical bifurcation of solitary waves is found at

$$
\kappa=\kappa_{c} \approx 0.01247946
$$


The power diagram of this bifurcation is shown in Fig. 6(b). We see that two smooth solution branches, namely the upper $c_{1}-c_{2}$ branch and the lower $d_{1}-d_{2}$ branch, tangentially connect at the bifurcation point $\left(\mu_{0}, P_{0}\right) \approx(3.28,14.35)$. Profiles of solitary waves at the marked $c_{1}, c_{2}, d_{1}, d_{2}$ locations on this power diagram are displayed in Fig. 6(c-d). Notice that these solutions are close to each other since the $c_{1}, c_{2}, d_{1}, d_{2}$ locations are near the bifurcation point $\left(\mu_{0}, P_{0}\right)$. As $\mu$ approaches this bifurcation point, we find that these solitary waves along both the lower and upper power branches approach each other, confirming that this is a transcritical bifurcation. Notice that the power diagram in Fig. 6(b) agrees with the analytical power formula (3.10) of transcritical bifurcations (see also the schematic power diagram in Fig. 1(c)). In addition, we have checked the conditions of transcritical bifurcations in Theorem 1 (Case 3), and found them satisfied here as well.
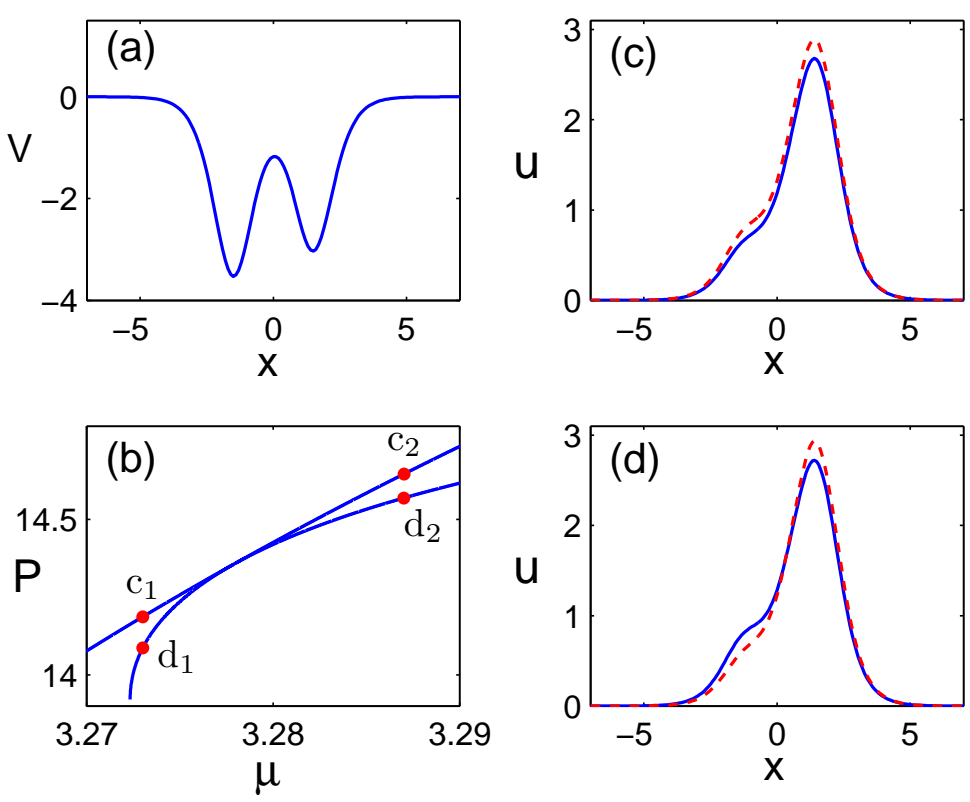

Figure 6: Transcritical bifurcation in Example 3 (see Eq. (5.7)). (a) The asymmetric double-well potential $V(x)$ in this example; (b) the power diagram; (c) profiles of solitary waves at locations $c_{1}$ (solid blue) and $c_{2}$ (dashed red) of the upper power curve in (b); (d) profiles of solitary waves at locations $d_{1}$ (solid blue) and $d_{2}$ (dashed red) of the lower power curve in (b).

What would happen to the bifurcations in the above three examples if the potential or the nonlinearity in those underlying GNLS equations is slightly perturbed? We have numerically studied this question and found that in Example 1, when the nonlinearity or the potential is slightly and arbitrarily perturbed (including perturbations to make the double-well potential (5.2) asymmetric), the saddle-node bifurcations (at point ' $\mathrm{C}$ ' of the symmetric-soliton branch and two others on the asymmetric-soliton branch in Fig. 2) always persist. For the two pitchfork bifurcations in this example (at points 'A,B' of Fig. 2), if the perturbed potential is still symmetric, then these pitchfork bifurcations would survive; but if the perturbed potential becomes asymmetric, then these pitchfork bifurcations are destroyed. In Example 2, the four saddle-node bifurcations on the power loop of Fig. 5 always persist under weak perturbations in the nonlinearity or the potential. In Example 3, the transcritical bifurcation is extremely sensitive and is destroyed under generic small 
perturbations to the system (such as when $\kappa \neq \kappa_{c}$ ). From these numerical results, we conclude that saddle-node bifurcations are generic and robust under weak perturbations to the system; pitchfork bifurcations are generally reliant on a symmetric potential; and transcritical bifurcations are very fragile and generally disappear under perturbations. These behaviors are consistent with similar statements on these bifurcations (based on the bifurcation conditions) below Theorem 1.

\section{Summary and discussion}

In this paper, we classified solitary wave bifurcations in the generalized NLS equations (2.1) with arbitrary nonlinearities and external potentials in arbitrary spatial dimensions. Sufficient analytical conditions were derived for three major types of solitary wave bifurcations, namely saddle-node bifurcations, pitchfork bifurcations and transcritical bifurcations. These conditions show that the generic solitary wave bifurcation is the saddle-node bifurcation; the pitchfork bifurcation generally requires certain symmetry conditions; and the transcritical bifurcation is rare. For these bifurcations, shapes of power diagrams near the bifurcation points were also obtained. It was shown that the power diagram for a pitchfork bifurcation exhibits double branching rather than the familiar triple branching, and the power diagram for a transcritical bifurcation features two curves tangentially touching each other rather than the familiar ' $x$ '-crossing. Numerical examples for these three types of bifurcations were presented as well. These examples show novel features such as power loops and double pitchfork bifurcations. The example of transcritical bifurcation seems to be the first report of such bifurcation in the generalized NLS equations (2.1).

The results in this paper are important not only for a general classification and understanding of solitary wave bifurcations in the generalized NLS equations (2.1). More importantly, the bifurcation conditions in Theorem 1 will be the basis for a general treatment of linear stability of solitary waves near bifurcation points. This stability analysis lies outside the scope of the present article and will be reported elsewhere. We do want to say here that the stability properties of solitary waves near bifurcation points in Eq. (2.1) show some important qualitative differences from those in finite-dimensional dynamical systems [25]. Details will be presented in a forthcoming article.

\section{Acknowledgment}

This work is supported in part by the Air Force Office of Scientific Research (Grant USAF 955009-1-0228) and the National Science Foundation (Grant DMS-0908167).

\section{References}

[1] Y. S. KIVSHAR and G. P. AGRAWAL, Optical Solitons: From Fibers to Photonic Crystals (Academic Press, San Diego, 2003).

[2] J. YANG, Nonlinear Waves in Integrable and Nonintegrable Systems (SIAM, Philadelphia, 2010). 
[3] B. BUFFONI, A. R. CHAMPNEYS, and J. F. TOLAND, Bifurcation and coalescence of a plethora of homoclinic orbits for a Hamiltonian system, J. Dyn. Differ. Equ. 8, 221-279 (1996).

[4] J. YANG, Classification of the solitary waves in coupled nonlinear Schroedinger equations, Physica D, 108, 92-112 (1997).

[5] T.S. YANG and T.R. AKYLAS, On asymmetric gravitycapillary solitary waves, J. Fluid Mech. 30, 215-232 (1997).

[6] M. CHEN, Solitary-wave and multi-pulsed travelling-wave solutions of Boussinesq systems, Appl. Anal. 75, 213-240 (2000).

[7] K.Y. KOlOSSOVSKI, A. V. BURYAK, V. V. STEBlinA, A. R. CHAMPNEYS, and R. A. SAMMUT, Higher-order nonlinear modes and bifurcation phenomena due to degenerate parametric four-wave mixing, Phys. Rev. E 62, 4309-4317 (2000).

[8] G. HERring, P. G. KEVREKIDiS, R. CARRETERO-GONZÁlEZ, B. A. MALOMED, D. J. FRANTZESKAKIS, and A. R. BISHOP, Trapped bright matter-wave solitons in the presence of localized inhomogeneities, Phys. Lett. A 345, 144-153 (2005).

[9] T. KAPITUlA, P. G. KEVREKIDIS, and Z. CHEN, Three is a crowd: Solitary waves in photorefractive media with three potential wells, SIAM J. Appl. Dyn. Syst. 5, 598-633 (2006).

[10] J. BURKE and E. KNOBLOCH, Homoclinic snaking: Structure and stability, Chaos 17, 037102 (2007).

[11] T. R. AKYLAS, G. HWANG, and J. YANG, From nonlocal gap solitary waves to bound states in periodic media, Proc. Roy. Soc. A 468, 116-135 (2012).

[12] A. ANKIEWICZA, N. AKHMEDIEV, and J. M. SOTO-CRESPO, Novel bifurcation phenomena for solitons in nonlinear saturable couplers, Optics Communications 116, 411-415 (1995).

[13] R. K. JACKSON and M. I. WEINSTEIN, Geometric analysis of bifurcation and symmetry breaking in a Gross-Pitaevskii equation, J. Statist. Phys. 116, 881-905 (2004).

[14] P. G. KEVREKIDIS, Z. CHEN, B. A. MALOMED, D. J. FRANTZESKAKIS, and M. I. WEINSTEIN, Spontaneous symmetry breaking in photonic lattices: Theory and experiment, Phys. Lett. A 340, 275-280 (2005).

[15] E. W. KIRR, P. G. KEVREKIDIS, E. SHLIZERMAN, and M. I. WEINSTEIN, Symmetrybreaking bifurcation in nonlinear Schrödinger/Gross-Pitaevskii equations, SIAM J. Math. Anal. 40, 566-604 (2008).

[16] A. SACCHETTI, Universal critical power for nonlinear Schrodinger equations with symmetric double well potential, Phys. Rev. Lett. 103, 194101 (2009).

[17] C. WANG, G. THEOCHARIS, P.G. KEVREKIDIS, N. WHITAKER, K.J.H. LAW, D.J. FRANTZESKAKIS, and B.A. MALOMED, Two-dimensional paradigm for symmetry breaking: The nonlinear Schrödinger equation with a four-well potential, Phys. Rev. E 80, 046611 (2009).

[18] E.W. KIRR, P.G. KEVREKIDIS, and D.E. PELINOVSKY, Symmetry-breaking bifurcation in the nonlinear Schrodinger equation with symmetric potentials, Commun. Math. Phys. 308, 795-844 (2011).

[19] M. MATUSZEWSKI, B. A. MALOMED, and M. TRIPPENBACH, Spontaneous symmetry breaking of solitons trapped in a double-channel potential, Phys. Rev. A 75: 063621 (2007).

[20] L. P. PITAEVSKII and S. STRINGARI, Bose-Einstein Condensation (Oxford University Press, Oxford, 2003).

[21] Y. POMEAU, A. RAMANI, and B. GRAMMATICOS, Structural stability of the Kortewegde 
Vries solitons under a singular perturbation, Physica D 31, 127-134 (1988).

[22] R. GRIMSHAW, Weakly nonlocal solitary waves in a singularly perturbed nonlinear Schrödinger equation, Stud. Appl. Math. 94, 257-270 (1995).

[23] D.C. CALVO and T.R. AKYLAS, On the formation of bound states by interacting nonlocal solitary waves, Physica D 101, 270-288 (1997).

[24] J. MURDOCK, Normal forms and unfoldings for local dynamical systems (Springer-Verlag, New York, 2003).

[25] J. GUCKENHEIMER and P. HOLMES, Nonlinear Oscillations, Dynamical Systems, and Bifurcations of Vector Fields (Springer-Verlag, New York, 1990).

[26] D. N. CHRISTODOULIDES and M. I. CARVALHO, Bright, dark, and gray spatial soliton states in photorefractive media, J. Opt. Soc. Am. B 12, 1628-1633 (1995).

[27] I.V. BARASHENKOV and E.V. ZEMLYANAYA, Travelling solitons in the externally driven nonlinear Schrödinger equation, J. Phys. A. 44465211 (2011). 